\title{
Mutational Analysis of EXT1in a Chinese Family Affected by Hereditary Multiple Osteochondroma
}

\author{
Guangzhi Yuan $\mathbb{D}^{1},{ }^{1}$ Qiang Su, ${ }^{2}$ Wenjun Liao, ${ }^{3}$ Wei Hou, ${ }^{4}$ Linke Huang, ${ }^{1}$ Peng Wang $\mathbb{D},{ }^{5}$ \\ and Huayu $\mathrm{Wu} \mathbb{1}^{4}$ \\ ${ }^{1}$ Orthopedics Department, The Second Affiliated Hospital of Guangxi Medical University, Nanning, Guangxi, China \\ ${ }^{2}$ Guangxi Institute for Food and Drug Control, Guangxi, China \\ ${ }^{3}$ Department of Cell Biology and Genetics, Guangxi Medical University, Nanning, Guangxi, China \\ ${ }^{4}$ Department of Physical Examination/Health Management Department, First Affiliated Hospital of Guangxi Medical University, \\ Nanning, Guangxi, China \\ ${ }^{5}$ Key Laboratory of Ministry of Education for Gastrointestinal Cancer, School of Basic Medical Sciences, Fujian Medical University, \\ Fuzhou, Fujian, China
}

Correspondence should be addressed to Peng Wang; wp33688@126.com and Huayu Wu; wuhuayu@gxmu.edu.cn

Received 17 September 2020; Accepted 8 July 2021; Published 9 August 2021

Academic Editor: Paul Harrison

Copyright ( 2021 Guangzhi Yuan et al. This is an open access article distributed under the Creative Commons Attribution License, which permits unrestricted use, distribution, and reproduction in any medium, provided the original work is properly cited.

\begin{abstract}
Objectives. To discuss the mutational features and their relationships with disease in a family with hereditary multiple osteochondroma (HMO) from Guangxi Province (GXBB-1 family), China. Methods. Genomic DNA and total mRNA were extracted from peripheral blood cells of GXBB-1 family members. Whole elements of the EXT1gene and its transcript, including exons, introns, exon-intron boundaries, and coding sequence (CDS) clones, were amplified and sequenced. Allele-specific PCR was used to confirm the position and type of mutation. Results. All patients from the GXBB-1 family harbored the cosegregating heterozygous c.1056+1G $>$ A mutation located in EXT1at an exon-intron boundary. Another three single-nucleotide polymorphisms (SNPs) were also detected in the patients, including IVS2+1G $>$ A in intron 2, c.1844 T>C [p.Pro (CCT) 614Pro (CCC)] in exon 3, and c.2534G>A [p.Glu (GAG) 844Glu (GAA)] in exon 9. The latter two SNPs were synonymous variations. Conclusions. The heterozygous c.1056+1G>A mutation cosegregated with the phenotype, indicating that it is athogenic mutation in the GXBB-1 family. This mutation is reported for the first time in Chinese HMO patients. IVS $2+1 \mathrm{G}>\mathrm{A}$ and c. $2534 \mathrm{G}>\mathrm{A}$ have no relationship with the occurrence of disease. However, c.1844 T>C and c.1056+1G $>\mathrm{A}$ are linked, and their interaction needs to be further studied. c.1844T $>C$ is a new SNP that has not been reported internationally.
\end{abstract}

\section{Introduction}

Hereditary multiple osteochondromas (HMOs), previously called hereditary multiple exostoses (HMEs), are rare autosomal dominant developmental bone disorders that usually affect cartilage ossification and are characterized by multiple benign osteocartilaginous masses that grow outward from the metaphyses of long bones (exostosis cartilaginea), such as the ends of femurs and tibias in the lower limbs or the humerus and forearm of the upper limbs [1]. HMO patients often have shortened stature, bony deformities, and restricted joint motion. Nearly all HMO patients harbor pathogenic mutations in the EXT gene family. Approximately 56-78\% and $21-44 \%$ of cases are caused by genetic mutations in EXT1 and EXT2, respectively [2]. However, the mutational frequencies in these two genes vary among ethnic groups. In the Western population, mutations in the EXT1 and EXT2 genes are responsible for $40 \% \sim 75 \%$ and $20 \%-40 \%$ of HMO cases, respectively [3-5], compared with $14 \sim 53 \%$ and $33 \% \sim 40 \%$ in the Chinese population, respectively $[6,7]$.

Boyer described the first HMO patient in 1814. As of 2008, 157 and 71 mutations in EXT1 and EXT2 related to HMO had been reported, respectively. By 2011, the corresponding numbers had increased to 170 and 84 , respectively. By 2013 and 2018, the numbers had increased to 429 and 223 and to 463 and 230 , respectively [8-10]. 


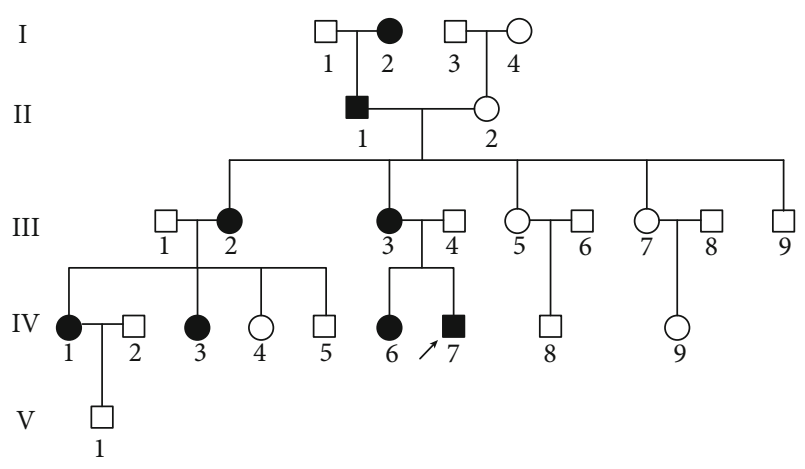

Figure 1: The Pedigree of the GXBB-1family with multiple hereditary osteochondromas from Guangxi Province. The proband (IV-7) is noted with a black arrow. $\mathbf{\square}$ : affected male; $\bullet$ affected female; $\square$ : healthy male; $\bigcirc$ : healthy female.

We performed genetic analysis in a four-generation HMO family from Guangxi Province to clarify the clinical features, pathogenic mutations, and mutation types and to accumulate more information for the diagnosis and treatment of HMO.

\section{Subjects and Methods}

2.1. Subjects. The proband (IV-7) was a ten-year-old boy in a multiple generation HMO family from the eastern region of Guangxi Province, China (GXBB-1). The distal ulna and radius, distal femur, and proximal tibia were observed to have multiple exostoses, and pathological diagnosis supported osteochondroma in this boy. All the other members of the GXBB-1 family were also examined according to International Diagnostic Standards in Figure 1 [3].

2.2. Mutation Screening. Genomic DNA and RNA were extracted from peripheral blood samples according to the manufacturer's instructions. cDNA was obtained by reverse transcription using RNA as a template. The coding sequence (CDS) of EXT1 was amplified using the following two primer pairs: EXT1aH-1223F/EXT1aH-1223R (5'-AGCTGAAAG TGTTGATTGGGA-3 ${ }^{\prime} / 5^{\prime}$-CTCATCGCCTATGACG GCAG-3') and EXT1bH-1293F/EXT1bH-1293F (5' -TTGG GTCCTTCAGATTCCTGG-3' $15^{\prime}$-TGGATCTGCACTGG

GAAGAGA-3 ${ }^{\prime}$ ). The amplification consisted of the following protocol: predenaturation for $4 \mathrm{~min}$ at $95^{\circ} \mathrm{C}$ followed by 30 cycles of denaturation at $94^{\circ} \mathrm{C}$ for $40 \mathrm{~s}$, annealing for $50 \mathrm{~s}$ at $58^{\circ} \mathrm{C}$, and extension for $5 \mathrm{~min}$ at $72^{\circ} \mathrm{C}$; and finally, extension at $72^{\circ} \mathrm{C}$ for $7 \mathrm{~min}$. The PCR products were purified, cloned, and sequenced at the Beijing Genomics Institute (BGI), Shenzhen.

Based on the CDS results for the EXT1 gene and the sequence of this gene (GenBank: NC_000008.11), the target gene fragment was amplified by PCR using EXT1-649F (GCAAAGACTGGGCAAACCAA) and EXT1-649R (AGG CCAAGCTGGCAATTAGAT). PCR programs included an initial denaturation of $5 \mathrm{~min}$ at $95^{\circ} \mathrm{C}$, followed by 30 cycles of $40 \mathrm{~s}$ at $94^{\circ} \mathrm{C}, 50 \mathrm{~s}$ at $57.5^{\circ} \mathrm{C}$, and $1 \mathrm{~min}$ at $72^{\circ} \mathrm{C}$. The PCR products were sequenced at the Beijing Genomics Institute
(BGI), Shenzhen. To further identify the mutation in the EXT1 gene in the family, allele-specific PCR (AS-PCR) was performed using the EXT1-402F (5-'GGAAGCAAAGAC TGGGCAAAC- $\left.3^{\prime}\right)$ and EXT1-402R (5'-AAGGCTCCAGG GCCTCTTAT- $3^{\prime}$ ) primers. PCR programs included an initial denaturation of $4 \mathrm{~min}$ at $95^{\circ} \mathrm{C}$, followed by 30 cycles of $40 \mathrm{~s}$ at $94^{\circ} \mathrm{C}, 50 \mathrm{~s}$ at $66^{\circ} \mathrm{C}$, and $55 \mathrm{~s}$ at $72^{\circ} \mathrm{C}$. PCR products were separated on a $1.5 \%$ polyacrylamide gel. Bands with a size of 402 bp were considered to have EXT1 mutations.

\section{Results}

3.1. Sanger Sequencing of the EXT1 Gene CDS Clones. All patients harbored a heterozygous deletion in the second exon of the EXT1 gene (NM_000127.2:c.1736-1829del), with only $87 \%$ alignment with reference sequences from the database (GenBank: NC_000008.11). This provided evidence that the patients had exon 2 skipping. Unaffected family members and normal controls had no deletions, resulting in $99 \%$ matching with the reference sequence.

3.2. Sanger Sequencing of the EXT1 Gene. As shown in Figure 2, all patients from the GXBB-1 family harbored a heterozygous splicing mutation (c.1056 G>A+1). The following three additional variations were also detected: (1) IVS2 $+1 \mathrm{G}>\mathrm{A}$ in intron 2, (2) c.1844 T >C [p.Pro (CCT) 614Pro (CCC)] in exon 3, and (3) c.2534G > A [p.Glu (GAG) 844Glu (GAA)] in exon 9. The latter two SNPs were synonymous variations.

3.3. Allele-Specific PCR. The $3^{\prime}$ AS primer perfectly matched only the mutant allele. Therefore, a $402 \mathrm{bp}$ fragment was amplified in the patients (II1, III3, IV6, III2, IV1, and IV3), while no products were detected in unaffected or normal controls.

3.4. Amino Acid Sequences of Wild-Type and Mutant Proteins. The original splicing site was abolished due to substitution of the first base $G$ with base $A$ in intron 2, which led to the exclusion of exon 2 in mature mRNA and premature termination at the protein level. We inferred that the truncated protein possessed 326 amino acids (p.Leu322TyrfsX6), with the last 5 amino acids being newly formed in Figure 3.

\section{Discussion}

HMO is highly genetically heterogeneous. The types and frequencies of mutations vary widely among ethnic groups, but the majority are missense mutations, nonsense mutations, splicing-change mutations, or small insertions or deletions [1]. Meanwhile, mutation hotspots occur, such as 1469delT in exon 6 and c.668G_C (p.R223P) in the EXT2 gene [5, 11, 12] and p.Leu490Argfs $* 9$ and p.Arg340Leu in the EXT1 gene $[13,14]$. George A. suggested that the $11^{\text {th }}$ exon of EXT1 was a mutation hotspot [6], and Zhuang reported three hotspot sequences prone to deletion residing in the $8^{\text {th }}$ exon. SCL found two novel mutation hotspots [5], namely, p.Ala409Profs $* 26$ and p.Ser290Ter and the highest frequency mutation p.Leu490Argfs $* 9$ in Brazilian HMO patients. The Brazilian population is a mixed population 
EXON2

EXON3

EXON1

EXON3
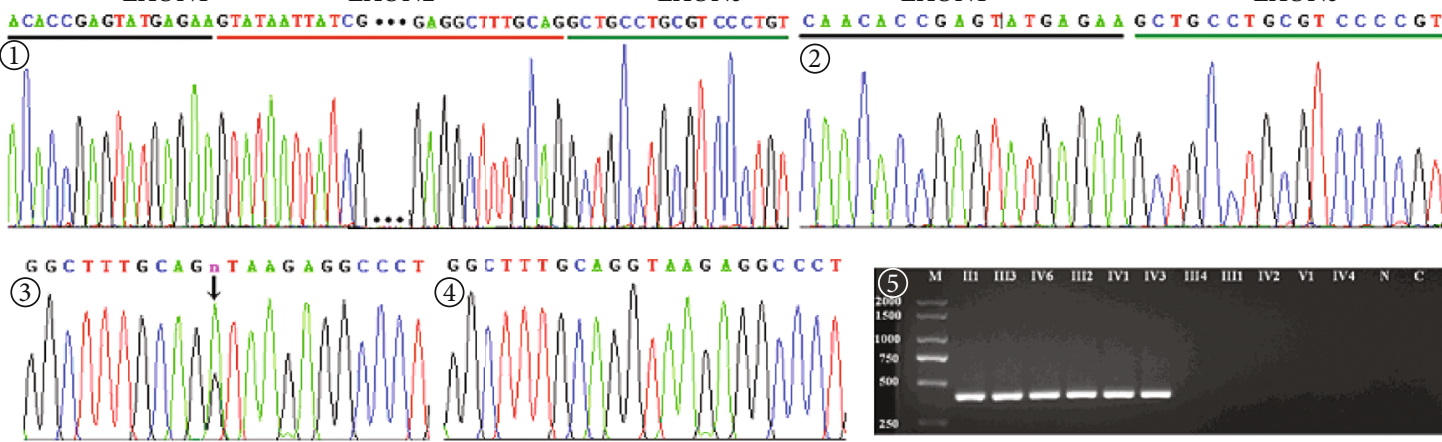

Figure 2: (1) III4 (unaffected), no exon deletion. (2) IV6 (affected), exon 2 deletion. (3) IV6 c.1056+1G > T in EXT1. (4) III4, no frame-shift mutation. (5) Allele-specific PCR: affected (II1, III3, IV6, III2, IV1, IV3), unaffected (III4, III1, IV2, V1, IV4), and normal control (N, C).

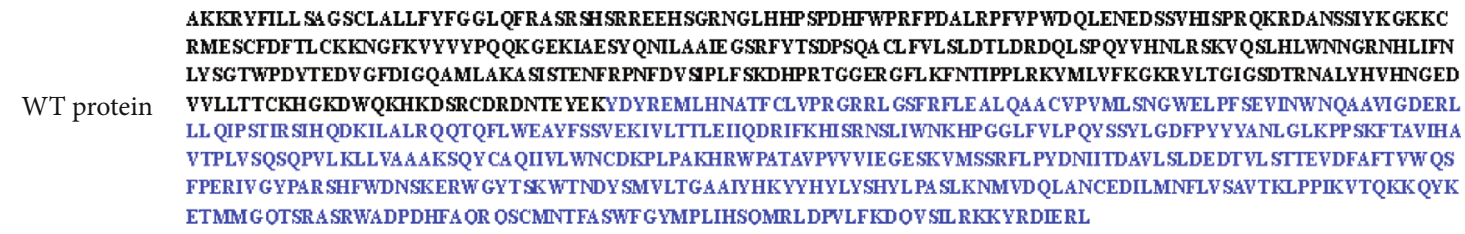

AKKR YFILL SA GSCLALLFYFG GL OFRASR SHSRREEHSGRNGLHHP SPDHFWPRFPDALRPFVPWDQLENEDSSVHISPR QKRDANSSIYKGKKC 作 VVLLTTCKH GKDWOKHKDSRCDRDNTE YE KYDYREMLHNATF CL VPR GRRL GSFRFLEALOAA CVPVML SNG WEL PF SEVINW NOAAVI GDERL 作 FPERIV GYPAR SHFWDNSKERW GYTSK WTNDY SMVLTGAATYHKYYHYLYSHYL PA SLKNMVDQLANCEDILMNFLV SAVTKLPPIKVTQKKQYK ETMM GOTSR ASR WADPDHFAOR OSCMITFA SWF G YMPLIHSOMRL DPVLFKDOV SIL RKKYRDIERL

$\begin{array}{llllllll}\mathbf{E} & \mathbf{K} & \mathrm{L} & \mathrm{P} & \mathrm{A} & \mathrm{S} & \mathrm{L} \text { Stop codon }\end{array}$

c. $1056+1 \mathrm{G}>\mathrm{A}$

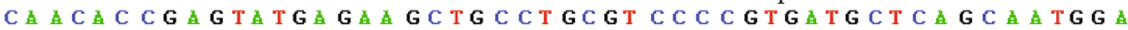
AKKRYFILL SA GSCLALLFYFG GLQFRASR SHSRREEH SGRNGLHHPSPDHFWPRFPDALRPFVPWDQLENEDSSVHISPR QKRDANSSTYKGKKC
RMESCFDFTLCKKNGFKVYYYPOOKGEKIAESY ONILAAIE GSRFYTSDP SQA CL FYLSLDTLDRDQLSPQYWHNLR SKY QSLHLWNNGRNHLIFN LY SGTWPDYTEDV GFDIGQAMLAKA SI STENFR RNFDVSPLF SKDHPRT GGER GFL KFNTIPPLRKYMLVFKGKR YLTGIGSDTRNALYHVHNGED VVLLTTCKHGKDWOKHKDSRCDRDNTEYEKL PASSL

FIGURE 3: Amino acid sequences of wild-type and mutant proteins. The length of the wild protein is 746aa, while the mutant truncated protein is 326aa (p.Leu322TyrfsX6). The red sequences (LPASL) indicate new amino acids caused by the frame shift.

with a strong influence of Europeans in its colonization history, which could be responsible for the presence of recurrent alterations in hotspots in HMO patients from Brazil [15].

Nonsense, frameshift, and splicing-site mutations can directly or indirectly cause premature protein translation, finally leading to rapid protein degeneration or loss of function. However, missense mutations that change specific or several amino acids and impair protein stability or normal function affect the corresponding biosynthesis process and lead to abnormal chondrocyte proliferation.

In this report, the splicing mutation $(\mathrm{c} .1056+1 \mathrm{G}>\mathrm{A})$ was found to substitute the GA at the $5^{\prime}$ splicing donor site with AA, while the $3^{\prime}$ acceptor site remained unchanged. This led to removal of the entire $2^{\text {nd }}$ exon and intron after mRNA splicing, forming the truncated protein p.Leu322TyrfsX6. Compared with the wild-type protein, the mutant protein had fewer (420) amino acids. Both of the proteins shared the $1^{\text {st }} \sim 321^{\text {st }}$ amino acids, but the $322^{\text {nd }}$ to $326^{\text {th }}$ amino acids were different (Figure 3).

The EXT1 protein contains four structural or functional domains, namely, signalP, transmembrane regions, exostosin (110 396 amino acids), and glycosyl transferase 64 (480 727 amino acids), which are very important for maintaining protein activities. The molecular mechanism underlying missense mutational effects on HMO phenotypes remains unclear, but the mechanisms for the other three types of mutations (nonsense, frameshift, and splicing-site mutations) seem obvious because all the mutations lead to partial or total removal of the exostosin and/or glycosyl transferase 64 protein domain [16, 17]. In our GXBB-1 family, the splicing-site substitution (c.1056+1G $>$ A) resulted in partial and total removal of the exostosin and glycosyl transferase 64 domains, respectively. The coding product of EXT1 and EXT2, i.e., heparan sulfate synthetase (HS), is a type II transmembrane protein crossing the surface of the endoplasmic reticulum. HS has glycosyltransferase activity and participates in the biosynthesis of heparan sulfate, which is crucial for normal skeletal development. EXT1 and EXT2 can form a protein heterodimer that has significantly higher biological activity than either of the proteins alone $[18,19]$. The c.1056+1G > A mutation in the EXT1 gene impairs the formation of an active heterodimer with EXT2, and this mutation cosegregated in the GXBB-1 family members. 
In the past few years, many novel mutations in the EXT gene have been reported. In 2016, Daichi et al. found 22 novel mutations in the EXT1 and EXT2 genes in Japanese HMO families [20]. One year later, SCL et al. observed 31 novel mutations in Brazilian HMO families [16]. In China, Li et al. reported 12 novel mutations in EXT1 and 14 novel mutations in EXT2 among $73 \mathrm{HMO}$ patients [8].

Deletions in EXT1 are the most common mutation type, followed by many nonsense and missense mutations, but splicing mutations are relatively rare in Chinese patients. Only c. $1417+1 \mathrm{G}>\mathrm{A}$, IVS5+1 G > A, IVS8+2 T > G, c.1883+ $1 \mathrm{G}>\mathrm{A}, \mathrm{c} . \mathrm{I} 8+2 \mathrm{~T}>\mathrm{G}-8, \mathrm{c} .1883+1 \mathrm{G}>\mathrm{A}-9$, and c. $1883+2 \mathrm{~T}>\mathrm{A}$ have been reported. The splicing mutation c.1056+1G $>A$ was first reported in the Chinese population. A few families with the same mutations have also been reported/recorded in the United Kingdom, Belgium, and Brazil. Therefore, we do not consider this to be a common or hotspot mutation $[4,16,21,22]$.

The IVS2+1G $>$ A variation in the GXBB-1 family is located in the EXT1 intron rather than at a splicing site. The c.2534G > A variation has no apparent effects on osteochondroma. The IVS2+1G $>$ A and c. $2534 \mathrm{G}>\mathrm{A}$ variations have been previously reported. c. $1844 \mathrm{~T}>\mathrm{C}$, another synonymous variation located in the EXT1 exon, is linked with the pathogenic mutation c. $1056+1 \mathrm{G}>\mathrm{A}$. Whether these variations interact remains to be further investigated.

\section{Data Availability}

The data used to support the findings of this study are included within the article. If additional details are required, please contact the corresponding author.

\section{Consent}

Not applicable.

\section{Conflicts of Interest}

The authors declare no conflicts of interest, financial, or otherwise.

\section{Authors' Contributions}

Guangzhi Yuan and QiangSu contributed equally to this work and should be considered co-first authors.

\section{Acknowledgments}

This work was funded by the Youth Foundation of Guangxi Medical University (GXMUYSF201802), the Specialized Programme of the Genetic Laboratory of Guangxi Medical University (2010-459), and the Guangxi Key Laboratory of Thalassemia Research (16-380-34).

\section{References}

[1] W. Wuyts and W. Van Hul, "Molecular basis of multiple exostoses: mutations in the EXT1 and EXT2 genes," Human Mutation, vol. 15 , no. 3 , pp. 220-227, 2000.
[2] E. Pedrini, I. Jennes, M. Tremosini et al., "Genotype-phenotype correlation study in 529 patients with multiple hereditary exostoses: identification of "protective" and "Risk" factors," The Journal of Bone and Joint Surgery. American Volume, vol. 93, no. 24, pp. 2294-2302, 1993.

[3] I. Jennes, M. M. Entius, E. Van Hul, A. Parra, L. Sangiorgi, and W. Wuyts, "Mutation screening of EXT1 and EXT2 by denaturing high-performance liquid chromatography, direct sequencing analysis, fluorescence in situ hybridization, and a new multiplex ligation-dependent probe amplification probe set in patients with multiple osteochondromas," The Journal of Molecular Diagnostics, vol. 10, no. 1, pp. 85-92, 2008.

[4] W. Heinritz, U. Hüffmeier, S. Strenge et al., "New mutations of EXT1 and EXT2 genes in German patients with multiple osteochondromas," Annals of Human Genetics, vol. 73, no. 3, pp. 283-291, 2009.

[5] C. Francannet, A. Cohen-Tanugi, M. Le Merrer, A. Munnich, J. Bonaventure, and L. Legeai-Mallet, "Genotype-phenotype correlation in hereditary multiple exostoses," Journal of Medical Genetics, vol. 38, no. 7, pp. 430-434, 2001.

[6] L. Xu, J. Xia, H. Jiang et al., "Mutation analysis of hereditary multiple exostoses in the Chinese," Human Genetics, vol. 105, no. 1-2, pp. 45-50, 1999.

[7] Y. Li, J. Wang, J. Tang et al., "Heterogeneous spectrum of EXT gene mutations in Chinese patients with hereditary multiple," Medicine, vol. 97, no. 42, 2018.

[8] X.-l. Guo, Y. Deng, and H.-g. Liu, "Clinical characteristics of hereditary multiple exostoses: a retrospective study of mainland Chinese cases in recent 23 years. Journal of Huazhong University of Science and Technology," Medical Science, vol. 34, pp. 42-50, 2014.

[9] F. A. N. G. Wei, P. E. I. Yuan-yuan, H. U. A. N. G. Wei-jun, H. U. Bin, and W. A. N. G. Yi-ming, "Investigation of polymorphisms in causal genes of HME:EXT1 and EXT2," JOURNAL OF SUN YAT-SEN UNIVERSITY (MEDICAL SCIENCES), vol. 32, no. 2, pp. 263-278, 2011.

[10] W. A. N. G. Wei, Q. I. U. Zheng-Qing, and S. O. N. G. HongMei, "A splicing mutation of EXT1 in a Chinese pedigree with hereditary multiple exostoses," Chin J ContempPediatr, vol. 16, no. 2, pp. 174-180, 2014.

[11] M. Ciavarella, M. Coco, F. Baorda et al., "20 novel point mutations and one large deletion in EXT1 and EXT2 genes: report of diagnostic screening in a large Italian cohort of patients affected by hereditary multiple exostosis," Gene, vol. 515, no. 2, pp. 339-348, 2013.

[12] G. A. Tanteles, M. Nicolaou, V. Neocleous et al., "Genetic screening of EXT1 and EXT2 in Cypriot families with hereditary multiple osteochondromas," Journal of Genetics, vol. 94, no. 4, pp. 749-754, 2015.

[13] L. Zhuang, S. D. Gerber, S. Kuchen, P. M. Villiger, and B. Trueb, "Deletion of exon 8 from the EXT1 gene causes multiple osteochondromas (MO) in a family with three affected members," SpringerPlus, vol. 5, no. 1, 2016.

[14] W. Wuyts, W. Van Hul, K. De Boulle et al., "Mutations in the EXT1 and EXT2 genes in hereditary multiple exostoses," American Journal of Human Genetics, vol. 62, no. 2, pp. 346354, 1998.

[15] S. C. L. Santos, I. M. P. O. Rizzo, R. I. Takata, C. E. SpeckMartins, J. M. Brum, and C. Sollaci, "Analysis of mutations in EXT1 and EXT2 in Brazilian patients with multiple osteochondromas," Molecular Genetics \& Genomic Medicine, vol. 6, no. 3, pp. 382-392, 2018. 
[16] Y. Pei, Y. Wang, W. Huang et al., "Novel mutations ofEXT1andEXT2Genes among families and sporadic cases with multiple exostoses," Genetic Testing and Molecular Biomarkers, vol. 14, no. 6, pp. 865-872, 2010.

[17] Y. Pei, Y. Wang, W. Huang et al., "Novel mutations of EXT1 and EXT2 genes among families and sporadic cases with multiple exostoses," Genetic Testing and Molecular Biomarkers, vol. 14, no. 6, pp. 865-872, 2010.

[18] B.-T. Kim, H. Kitagawa, J. Tanaka, J.-i. Tamura, and K. Sugahara, "In vitro heparan sulfate polymerization," The Journal of Biological Chemistry, vol. 278, no. 43, pp. 4161841623, 2003.

[19] B. Zak, "Hereditary multiple exostoses and heparan sulfate polymerization," Hereditary multiple exostoses and heparan sulfate polymerization, Biochimica et Biophysica Acta, vol. 1573, no. 3, pp. 346-355, 2002.

[20] D. Ishimaru, M. Gotoh, S. Takayama et al., "Large-scale mutational analysis in the EXT1 and EXT2 genes for Japanese patients with multiple osteochondromas," BMC Genetics, vol. 17, no. 1, 2016.

[21] F. U. Qi-hua, L. I. Yu-chan, W. A. N. G. Jian, and W. A. N. G. Jing, "Study on EXT gene mutation of a pedigree with hereditary multiple exostoses," J Diagn Concepts Pract, vol. 7, no. 6, pp. 625-627, 2008.

[22] L. Lonie, D. E. Porter, M. Fraser et al., "Determination of the mutation spectrum of the EXT1/EXT2 genes in British Caucasian patients with multiple osteochondromas, and exclusion of six candidate genes in EXT negative cases," Human Mutation, vol. 27, no. 11, p. 1160, 2006. 